\title{
PROPUESTA PARA LA INVESTIGACIÓN DE LA PERTINENCIA EDUCATIVA EN LA REGIÓN JUNÍN*
}

\author{
Palomino León, Juan de Dios y y Flores Arroyo, Rosario Inés²
}

Facultad de Pedagogía y Humanidades de la Universidad Nacional del Centro del Perú

\begin{abstract}
RESUMEN
La presente investigación se realizó con la intención de saber ¿Cómo sería un sistema problemático diseñado para investigar la pertinencia educativa en la Región Junín? Los objetivos fueron: General: Diseñar un sistema problemático para la investigación de la pertinencia educativa en la Región Junín.Específicos: Sistematizar la formulación teórica de la pertinencia educativa y sus connotaciones particulares para ámbitos como la Región Junín, y, Determinar en la realidad educativa de la Región Junín las dimensiones, áreas problemáticas y principales problemas relacionados con la pertinencia educativa susceptibles de ser abordados a través de la investigación pedagógica. La hipótesis fue: Un sistema problemático diseñado para investigar la pertinencia educativa en la Región Junín sería tal que partiendo de las características de los educandos orientara la búsqueda de la pertinencia hacia las diversas áreas del sistema y del proceso educativo, relacionándola con el contexto económico, social, cultural e histórico, en diversos espacios subregionales y considerando las exigencias nacionales y mundiales para la educación. Para comprobarla se recurrió a una metodología descriptiva, analítico crítica y constructiva. Los medios y materiales fueron propios de la investigación bibliográfica y el trabajo de gabinete.
\end{abstract}

Palabras clave: pertinencia educativa, Calidad educativa, Demandas educativas, Contextualización educativa, Centralidad del educando.

\section{PROPOSAL FOR THE EDUCATION RELEVANCE RESEARCH IN THE JUNIN REGION}

\begin{abstract}
The present research was conducted to know how would a problematic system be designed to research the education relevance in the Junín Region? The objectives were: General: Design a problematic system to research the educational relevance an its particular connotations to areas as the Junín Region, and, to determinate on the educational reality of the Junín Region, the dimensions, problematic areas and principal problems in relation with the educative relevance susceptible to be approached through the pedagogical research. The hypothesis was: A problematic system designed to research the educational relevance in the Junín Region would be such that starting from the students characteristics it will guide to the search of the relevance, toward diverse areas of the system and educational process, relating it with the economic, social, cultural and historical context, in diverse sub regional spaces and considering the national and World demands for education. To check, it was used a descriptive, analytic critic and constructive methodology. The means and materials were characteristic of the bibliographical investigation and the cabinet work.
\end{abstract}

\footnotetext{
* Este trabajo de investigación fue recibido el 20/12/2008 retornado para su revisión 26/10/2009 y aprobado para su publicación 16/11/2009

1Email: edmaore@yahoo.es

22Email: manuelortize@hotmail.com
} 


\section{INTRODUCCIÓN}

En el actual Proyecto Educativo Regional al 2021 de la Región Junín el problema factual de la falta de pertinencia educativa es destacado como el primer problema priorizado para abordarse; asimismo en el Proyecto Educativo Nacional al 2021 también se relieva como uno de los principales problemas de la realidad educativa peruana; éste problema fue advertido en Huancayo por el autor de la presente investigación en trabajos de fines de 1990, reiterado en una investigación del 2004 y nuevamente en el 2008 demostrando que la pertinencia educativa estaba fundamentalmente ligada a la búsqueda de la calidad educativa. No obstante, en la universidad y otros medios académicos y políticos el tema es tocado sólo eventualmente y no existe una clara comprensión de su significado, su contenido y sus implicancias; es más, muchos egresados de la educación superior no tienen nociones de ello, y, peor aún, en los propios documentos en que se reconoce la importancia de este problema, se encuentran imprecisiones, ambigüedades y hasta contradicciones; esto es natural cuando se parte de la captación intuitiva de una problemática factual pero faltando aún la investigación científica de la realidad; esto, deja un campo amplio e importante de necesario esclarecimiento para los profesionales de la educación e implica también la responsabilidad de los docentes universitarios en ella; en particular es necesario investigar la pertinencia educativa en el Perú y en sus Regiones, pero siendo la pertinencia un concepto polisémico, complejo, que abarca todos los ámbitos educativos, que por sí mismos son de harta complejidad y problemáticos, más aún si se considera la situación crítica de la educación peruana actual, entonces, la investigación de la pertinencia ofrece tales dificultades que es necesario ordenar el campo investigativo, y existen las posibilidades de hacerlo de acuerdo a las actuales condiciones epistémicas de la investigación; por ello el problema planteado para la investigación que aquí se presenta fue ¿Cómo sería un sistema problemático diseñado para investigar la pertinencia educativa en la Región Junín?

Por lo señalado, los objetivos del presente trabajo fueron: Diseñar un sistema problemático para la investigación de la pertinencia educativa en la Región Junín. Sistematizar la formulación teórica de la pertinencia educativa y sus connotaciones particulares para la Región Junín, las dimensiones, áreas problemáticas y principales problemas relacionados con la pertinencia educativa susceptibles de ser abordados a través de la investigación pedagógica. La hipótesis a demostrarse fue: un sistema problemático diseñado para investigar la pertinencia educativa en la Región Junín sería tal que partiendo de las características de los educandos orientara la búsqueda de la pertinencia hacia las diversas áreas del sistema y del proceso educativo, relacionándola con el contexto económico, social, cultural, e histórico, en diversos espacios subregionales y mundiales para la educación. Para la demostración se recurrió a una metodología descriptiva, analítico crítica y constructiva.

En el primer capítulo del presente trabajo se especifica el planteamiento del estudio, con el problema, los objetivos, la justificación y las limitaciones. En el segundo se desarrolla el marco teórico, los antecedentes, las bases epistémicas de la investigación se discute la pertinencia y sus connotaciones, su significado en el país y la región. En el capítulo tercero, se especifica el método y diseño del trabajo. En el capítulo cuarto, se presentan los resultados y se señalan perspectivas para un ulterior desarrollo. En el quinto capítulo se realizan breves constataciones y comentarios respecto a los resultados.

\section{MATERIAL Y MÉTODOS.}

Siendo la investigación de tipo sustantivo, teórica y de segundo nivel, los materiales usados fueron la bibliografía y la web grafía sobre los temas epistemológicos y metodológicos de la investigación educacional además de estudios realizados sobre aspectos contextuales de la realidad educativa de la Región Junín, se realizaron resúmenes, recensiones, y copias de textos utilizando las fichas respectivas. La metodología usada fue de tipo analítico crítico heurística y constructiva, con un diseño descriptivo, se partió de primero de determinar las bases epistémicas de los problemas y los sistemas de problemas, para luego analizar el concepto de pertinencia educativa, y determinar la estructura de su significación desde la óptica de los actuales avances de la teoría pedagógica, seguidamente se aplicaron las determinaciones sobre sistematización de problemas a la problemática de la pertinencia para determinar un sistema problemático, luego, se estudiaron algunos trabajos sobre la realidad contextual de la 
educación en la Región Junín y se buscó determinar el sistema de problemas sobre la pertinencia educativa.

\section{RESULTADOS}

Para organizar el sistema problemático se tuvo como base tres criterios generales: 1) Centralidad del educando, 2) Relación con la práctica y la transformación, 3) Partir de los aspectos más directamente relacionados a los aspectos más periféricos y por último los especiales. De acuerdo a ello, partiendo del problema fundamental: ¿Cómo lograr que la educación en la Región Junín corresponda adecuadamente a las condiciones, intereses, necesidades y posibilidades de los educandos? se logró sistematizar cuatro tipos de problemas:

1) PROBLEMAS DE DIAGNÓSTIC0: 1.1. ¿Cuáles son las condiciones de los educandos en la Región Junín en relación con su participación en el proceso educativo?, 1.2. ¿Cuáles son los intereses de los educandos de la Región Junín en relación con su participación en el proceso educativo?, 1.3. ¿Cuáles son las necesidades de los educandos de la Región Junín en relación con su participación en el proceso educativo?, 1.4. ¿Cuáles son las potencialidades de los educandos de la Región Junín en relación con su participación en el proceso educativo?

2) PROBLEMAS PRESCRIPTIVOS: 2.1. ¿Cuáles deberían ser las características de la educación en el sistema educativo de la Región Junín para lograr la consideración, respeto y trato a los educandos de acuerdo a sus condiciones?, 2.2. ¿Cuáles deberían ser las características de la educación en el sistema educativo de la Región Junín para que logren considerar, partir de y satisfacer los intereses de los educandos?, 2.3. ¿Cuáles deberían ser las características de la educación en el sistema educativo de la Región Junín para que logren orientar, y proporcionar a los educandos de los medios adquiribles por la educación para satisfacer sus necesidades en el marco de las condiciones, oportunidades y exigencias de la realidad?, 2.4. ¿Cuáles deberían ser las características de la educación en el sistema educativo de la Región Junín para que logren promover el desarrollo de las potencialidades del educando, optimizándolas, en el marco de las condiciones y oportunidades de la realidad?
3) PROBLEMAS EVALUATIVOS. 3.1. ¿En qué niveles, la educación que se realiza en el sistema educativo de la Región Junín considera, respeta y trata adecuadamente al educando de acuerdo a sus condiciones?, 3.2. ¿A qué niveles, la educación que se realiza en el sistema educativo de la Región Junín logra la consideración, el partir de, y satisfacer, los intereses de los educandos? 3.3. ¿A qué nivel, la educación que se realiza en el sistema educativo de la Región Junín logra orientar al educando y proporcionarle los medios adquiribles por la educación, para satisfacer sus necesidades en el marco a las condiciones, oportunidades y exigencias de la realidad? 3.4. ¿A qué nivel, la educación que se realiza en el sistema educativo de la Región Junín logra promover el desarrollo de las potencialidades del educando, optimizándolas, en el marco de las condiciones y oportunidades de la realidad?

4) PROBLEMAS DE INNOVACIÓN: 4.1. ¿La innovación "X", contribuirá efectivamente a mejorar, el respeto y trato adecuado al educando de acuerdo a sus condiciones, en el proceso educativo que se da en la Región Junín?, 4.2. ¿La innovación "X", contribuirá efectivamente a mejorar en la educación que se realiza en el sistema educativo de la Región Junín la consideración, el partir de, y satisfacer, los intereses de los educandos?, 4.3. ¿La innovación " $X$ " contribuirá efectivamente a mejorar la educación que se realiza en el sistema educativo de la Región Junín respecto a orientar al educando y proporcionarle los medios adquiribles por la educación, para satisfacer sus necesidades en el marco a las condiciones, oportunidades y exigencias de la realidad?, 4.4. ¿La innovación "X" contribuirá efectivamente a mejorar el desarrollo de las potencialidades del educando, optimizándolas, en el marco de las condiciones y oportunidades de la realidad, en la educación realizada en el sistema educativo de la Región Junín?

Se estipuló la conformación de un círculo virtuoso reiterando los cuatro tipos de problemas anteriores. Se determinaron orientaciones y ejemplos para la subdivisión de los problemas anteriores, y para su particularización. También se propuso una reorientación de las diversas direcciones de la investigación de la pertinencia educativa en función de tomar como eje la centralidad del educando. 


\section{DISCUSIÓN}

Se logró diseñar un sistema problemático coherente, que partiendo de las características de los educandos se orientó, en la búsqueda de la pertinencia, hacia las áreas del sistema y el proceso educativo, con relación a contextos del entorno educacional, pero no se lograron mayores precisiones en cuando a precisar las particularidades de diversas áreas del sistema y menos aún precisiones respecto a los espacios subregionales, también hizo falta comprender con mayor amplitud y precisión en la problemática, las exigencias nacionales y mundiales a la educación.

Por lo señalado puede indicarse que se logró verificar la hipótesis aunque en forma parcial, debido a los aspectos que aún quedan por desarrollar.También puede observarse que en el decurso del trabajo se ha logrado cumplir con el objetivo general de la investigación que fue "Diseñar un sistema problemático para la investigación de la pertinencia educativa en la Región Junín".

También se ha logrado Sistematizar la formulación teórica de la pertinencia educativa y ciertas connotaciones generales para la Región Junín, pero en este aspecto ha faltado el logro de particularizaciones. Cabe señalar que debido a la complejidad del fenómeno educativo, hasta hoy no existe consenso en lo que son sujetos, elementos, procesos, condiciones y otros componentes de la educación, por ello, se ha optado por una selección lo más inclusiva posible, pero que aún en desarrollo de los componentes de la educación, lo que ameritará mayores precisiones en el futuro. También se ha logrado, aunque parcialmente, determinar dimensiones, áreas y principales problemas de la pertinencia educativa posibles de ser investigados en la Región Junín.

Es de destacarse que la orientación fundamental de la sistematización de los problemas es la de transformar la realidad educativa vigente, pasando de investigaciones de tipo diagnóstico a las prescriptivas, evaluativas y de innovación considerándolas como un ciclo que da lugar a un círculo virtuoso en pro de lograr la pertinencia educativa.

Es notorio en los resultados que el análisis de la problemática y su aplicación en la educación resulta más amplio y profundo de lo que se ha logrado en la presente investigación, de manera que en cuanto a la particularización de los problemas queda aún largo camino por recorrer, y si se considera el dinamismo que tiene la realidad educativa, este camino se prolonga "ad infinitum".

También es necesario señalar que definir los significados y orientaciones de la investigación de la pertinencia tiene relación con los avances que se tengan sobre el estudio de la realidad concreta de los ámbitos geográficos, sociales, económicos y culturales en que la educación se desarrolla; y, si no existen estudios sistemáticos, abarcadores y profundos, sobre la realidad de Junín, esto limita la precisión y amplitud a que se pueda llegar en las orientaciones sobre la investigación de la pertinencia.

\section{CONCLUSIONES}

- Un sistema problemático diseñado para investigar la pertinencia educativa en la Región es tal que partiendo de las características de los educandos orienta la búsqueda de la pertinencia hacia las diversas áreas del sistema y del proceso educativo, relacionándola con el contexto económico, social, cultural e histórico.

- El concepto de pertinencia es relacional y bidireccional pues la educación cumple un rol de nexo entre las condiciones, intereses, necesidades y potencialidades del educando con las condiciones, demandas y exigencias de la realidad contextual de la educación.

- Las diversas connotaciones de la pertinencia educativa encuentran un ordenamiento adecuado a las actuales teorías pedagógicas, si considera como eje ordenador la centralidad del educando; en torno a él son susceptibles de formularse e interpretarse todas ellas.

- El estudio de la pertinencia educativa es concomitante con el estudio del educando por un lado y con el estudio de la realidad contextual en que se imparte la educación; de los avances en ambas áreas dependerá también el avance de los estudios de la pertinencia.

- Un programa de investigación que busque transformar la realidad puede pasar de etapas de investigación diagnóstica, a la de investigación prescriptiva, luego, evaluativa, y posteriormente innovadora, lo 
que puede cambiar la realidad y dar lugar a la repetición de las etapas en un círculo virtuoso de investigación.

\section{RECOMENDACIONES}

Priorizar la investigación de la pertinencia educativa en la Región y el País como uno de los problemas factuales de mayor importancia y urgencia demandados por la sociedad.

Profundizar los estudios sobre la pertinencia educativa en los ámbitos de formación superior considerando la necesidad de ordenar su orientación a partir del reconocimiento de la centralidad del educando en el proceso educativo.

Desarrollar investigaciones de tipo diagnóstico, prescriptivas, evaluativas e innovadoras respecto a la pertinencia educativa, tomando como fundamento la atención a las condiciones, intereses, necesidades y potencialidades del educando.

Ampliar, profundizar, precisar las orientaciones y líneas de investigación de la pertinencia educativa en las diferentes áreas y subprocesos del proceso educativo, precisando la problemática para espacios subregionales de la Región Junín.

\section{LITERATURA CITADA}

Anquín, A. 2006. Demanda de pertinencia a la política educativa, a la formación docente y a las prácticas docentes en la región. Universidad Nacional de Salta. Argentina.

Bertalanffy, L. 1995. Teoría general de los sistemas $10^{\circ}$. Fondo de Cultura Económica, México D.F.

Blanco, R. 2007. Educación de calidad para todos: un asunto de derechos humanos. OREALCUNESCO, Santiago - Chile.

Bourdieu, P.1975. El oficio del sociólogo. Siglo XXI, Buenos Aires.

Dirección Regional de Educación de Junín. 2008. Proyecto Educativo Regional de Junín al 2021. M.E. DREJ., Huancayo - Perú.
Lakatos, I. 2006. Escritos filosóficos. La metodología de los programas de investigación científica. En: Mazzi Huacaychuco, compilador. Epistemología. Lima: San Marcos; 2006. p.624 - 687. En revista iberoamericana de educación. www.rieoei.org/ deloslectores/972Mendez.pdf

Ortiz, F. 2008. Diccionario de metodología de la investigación científica. $2^{\mathrm{a}}$ ed. Editorial Limusa, México D.F.

Palomino, J. 1999. Principales problemas educacionales de la provincia de Huancayo. Ponencia presentada al II Congreso Nacional de Investigación Educativa del Perú, Nov. 1999 Huancayo - Perú.

Palomino, J. 2004. Los problemas más relevantes del sistema educativo en la Región Junín. Instituto de Investigación de la FPH - UNCP, Huancayo.

Palomino, J. 2008. La pertinencia educativa como factor de la calidad educativa. Instituto de Investigación de la FPH - UNCP, Huancayo.

Rojo, N. 1999. Pertinencia social de los programas académicos de perfil gerencial en el sector salud. En revista cubana de educación médica superior, V. 13 N 1. La Habana.

Sañudo, L. 2006. Pertinencia y calidad de la investigación educativa. Recomendaciones para la gestión. En Educar Rev. Del gobierno del Estado de Jalisco. http://educacion.jalisco.gob.mx

Silveira, S. 2006. Poniéndonos lentes de género para mejorar la calidad, la pertinencia y la equidad de las políticas de educación profesional y técnica. En Boletín Téc. SENAC. Río de Janeiro V. 32 N.2.

Silvio, J. 2006. Hacia una educación virtual de calidad, pero con equidad y pertinencia. En revista de universidad y sociedad del conocimiento Vol. $3 \mathrm{~N}^{0} 1$ 2006. Catalunya - España. www.uoc.edu/rusc

Tamayo, M. 1990. Diccionario de la investigación científica. $2^{\mathrm{a}}$ ed. $1^{\mathrm{a}}$ reimpresión, Editorial Limusa, México D.F.

Ticona, I. 2005. Construyendo participativamente una educación alternativa de jóvenes y adultos con identidad y pertinencia. CERFAL-CEE, La Paz - Bolivia. 